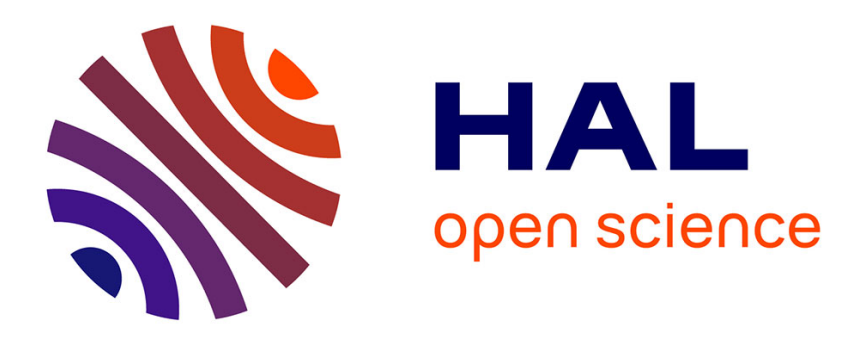

\title{
Order antimorphisms of finite-dimensional cones
}

Cormac Walsh

\section{To cite this version:}

Cormac Walsh. Order antimorphisms of finite-dimensional cones . Selecta Mathematica (New Series), 2020, 26 (4), paper number 53. hal-01673777v2

\section{HAL Id: hal-01673777 \\ https://hal.science/hal-01673777v2}

Submitted on 10 Jan 2018

HAL is a multi-disciplinary open access archive for the deposit and dissemination of scientific research documents, whether they are published or not. The documents may come from teaching and research institutions in France or abroad, or from public or private research centers.
L'archive ouverte pluridisciplinaire HAL, est destinée au dépôt et à la diffusion de documents scientifiques de niveau recherche, publiés ou non, émanant des établissements d'enseignement et de recherche français ou étrangers, des laboratoires publics ou privés. 


\title{
ORDER ANTIMORPHISMS OF FINITE-DIMENSIONAL CONES
}

\author{
CORMAC WALSH
}

\begin{abstract}
We show that an order antimorphism on a finite-dimensional cone having no one-dimensional factors is homogeneous of degree -1 . A consequence is that the existence of an order antimorphism on a finite-dimensional cone implies that the cone is a symmetric cone.
\end{abstract}

\section{INTRODUCTION}

Given an order structure, it is natural to study its isomorphisms and antimorphisms. Recall the former are the the bijective maps that preserve the order in both directions, and the latter are those that reverse the order in both directions.

The case of ordered vector spaces has received much attention, starting with Alexandrov and Ovchinnikova [1] and Zeeman [14], who studied maps preserving the light cone that arises in special relativity. This work has be extended to more general ordered vector spaces by Rothaus [12], Noll and Schäffer [10, 9], and Artstein-Avidan and Slomka [3]. One typically searches for conditions under which the isomorphisms are affine.

For example, in [10] it was shown that any order isomorphism between two proper open convex cones, one of which has no one-dimensional factors, in a finite dimensional linear space is the restriction of a linear isomorphism between the two cones. Here, proper means that the cone contains no complete lines, and having no one-dimensional factors means that it can not be written as the Cartesian product of $(0, \infty)$ with a cone of smaller dimension. The latter condition is obviously necessary - if there is a onedimensional factor then taking any non-linear order-preserving bijection on this factor and the identity on the complement gives an order isomorphism that is not linear.

Order antimorphisms are also interesting. In [2] the antimorphisms of the set of lowersemicontinuous convex functions on $\mathbb{R}^{d}$ were considered. It was shown that the only such map, up to linear terms, is the Legendre-Fenchel transform.

Here we investigate the antimorphisms of ordered vector spaces. If the domain is the whole space, then there is nothing new - the antimorphisms are just the isomorphisms composed with a reflection in the origin. But the existence of antimorphisms on open cones is more subtle. Our main result is that, when there are no one-dimensional factors, they must be antihomogeneous, that is, satisfy $\phi(\lambda x)=\lambda^{-1} \phi(x)$, for all elements $x$ of the cone, and and $\lambda>0$.

Theorem 1.1. Let $\phi: C \rightarrow C^{\prime}$ be an order antimorphism between two proper open convex cones in a finite-dimensional linear space. If $C$ has no one-dimensional factors, then $\phi$ is antihomogeneous.

In [13], it was shown that there can be an antihomogeneous order-antimorphism between two finite-dimensional cones if and only if both cones are symmetric, that is,

Date: January 10, 2018. 
homogeneous and self-dual. Combining this with the theorem above, we get the following.

Corollary 1.2. Let $\phi: C \rightarrow C^{\prime}$ be an order antimorphism between two proper open convex cones in a finite-dimensional linear space. Then $C$ and $C^{\prime}$ are symmetric cones.

The technique in [13] was to consider the Funk metric on the cone, which is a nonsymmetric metric defined using the order and homogeneity structures. Each antihomogeneous antimorphism on a cone reverses this metric [9]. So, it was fruitful to study the two horofunction boundaries of this metric, those in the forward and backward directions.

Since in this paper we do not assume antihomogeneity, the metric techniques do not work. Instead, we will use techniques similar to those in [12], [10], and [3]. Namely, we study how the map acts on line segments parallel to extreme rays of the cone. This means that our proof is essentially finite dimensional. Indeed, there are many interesting cones in infinite dimension that have few or no extreme rays.

A step in our proof may be of independent interest. We show that if the domain cone has a product structure then so does the image cone, and this product structure is respected by the order antimorphism.

Theorem 1.3. Let $\phi: C \rightarrow C^{\prime}$ be an order antimorphism between two proper open convex cones in a finite-dimensional linear space. Assume that $C$ has the decomposition $C=C_{1} \times \cdots \times C_{n}$. Then, $C^{\prime}$ has a decomposition $C^{\prime}=C_{1}^{\prime} \times \cdots \times C_{n}^{\prime}$, and $\phi$ takes the form

$$
\phi\left(x_{1}, \ldots, x_{n}\right)=\left(\phi_{1}\left(x_{1}\right), \ldots, \phi_{n}\left(x_{n}\right)\right), \quad \text { for all }\left(x_{1}, \ldots, x_{n}\right) \in C .
$$

It is also interesting to consider the infinite dimensional case. For isomorphisms, there are some results in [12] and [10]. The theory here is somewhat unsatisfactory however. A typical assumption is that the cone is the convex hull of its extreme rays, which is a very strong assumption in infinite dimension.

One may also try to find an infinite-dimensional version of Corollary 1.2. A natural generalisation of the concept of an ordered vector space is an order unit space. On the other hand, there is no straightforward generalisation of symmetric cones. However, one can consider the infinite-dimensional version of the Euclidean Jordan algebras to be the $J B$-algebras. In [8], the question was raised whether the existence of an antihomogeneous order antimorphism on an order-unit space implies that it is a JB-algebra? Corollary 1.2 suggests that the antihomogeneity assumption may not even be necessary.

\section{Preliminaries}

2.1. Isomorphisms and Antimorphisms. Let $C$ be an open convex cone in a real finite-dimensional vector space $V$. This means that $C$ is an open convex set that is invariant under multiplication by positive scalars. If $C$ contains no full lines, then $C$ is called a proper open convex cone.

The cone $C$ induces a partial order $\leq_{C}$ on $V$ as follows: $x \leq_{C} y$ if $y-x \in \operatorname{cl} C$. Here we are using cl to denote the closure of a set. The interior is denoted int. An order interval is a set of the form $[x, y]:=\left\{w \in C \mid x \leq_{C} w \leq_{C} y\right\}$.

Let $\phi: C \rightarrow C^{\prime}$ be a map between two proper open convex cones in $V$. We say that $\phi$ is isotone if $x \leq_{C} y$ implies $\phi(x) \leq_{C^{\prime}} \phi(y)$. If $\phi$ is a bijection and both it and its inverse are isotone, then we say that $\phi$ is an order isomorphism.

Likewise, $\phi$ is said to be antitone if $x \leq_{C} y$ implies $\phi(y) \leq_{C^{\prime}} \phi(x)$, and is said to be an order antimorphism if it is a bijection and it and its inverse are antitone. 
We say that $\phi$ is homogeneous of degree $\alpha \in \mathbb{R}$ if $\phi(\lambda x)=\lambda^{\alpha} \phi(x)$, for all $x \in C$ and $\lambda>0$. Maps that are homogeneous of degree -1 we call antihomogeneous, and maps that are homogeneous of degree 1 we just call homogeneous.

2.2. Product cones. Let $C_{1}, C_{2}$, and $C$ be non-empty convex cones in the linear space $V$. We say that $C$ is the direct product of $C_{1}$ and $C_{2}$ if $C=C_{1}+C_{2}$ and $\operatorname{lin} C_{1} \cap \operatorname{lin} C_{2}=$ $\{0\}$. Here lin denotes the linear span of a set. In this case we write $C=C_{1} \times C_{2}$.

If $C=C_{1} \times C_{2}$, then $\operatorname{lin} C$ is the (linear space) direct sum of $\operatorname{lin} C_{1}$ and $\operatorname{lin} C_{2}$.

A cone is said to be irreducible if it can not be written as a product. Given any proper open convex cone $C$, we can decompose it into a finite number of factors $C=C_{1} \times \cdots \times C_{n}$, each of which is irreducible. This decomposition is unique up to ordering of the factors. We say that $C$ has no one-dimensional factors if none of these factors are one-dimensional.

The order structure on a product cone is the product of the orders on the factors, that is, $\left(x_{1}, x_{2}\right) \leq_{C}\left(y_{1}, y_{2}\right)$ if and only if $x_{1} \leq_{C_{1}} y_{1}$ and $x_{2} \leq_{C_{2}} y_{2}$.

The following theorem was proved in [9]. Versions of this theorem with stronger assumptions appeared in [12] and [3].

Theorem 2.1. Let $\phi: C \rightarrow C^{\prime}$ be an order isomorphism between two proper open convex cones in a finite-dimensional linear space. If $C$ has no one-dimensional factors, then $\phi$ is the restriction to $C$ of a linear map.

2.3. Symmetric cones. A proper open convex cone $C$ in a real finite-dimensional vector space $V$ is called symmetric if it is homogeneous and self-dual. Recall that $C$ is homogeneous if its linear automorphism group $\operatorname{Aut}(C):=\{A \in \mathrm{GL}(V) \mid A(C)=C\}$ acts transitively on it, and it is self-dual if there exists an inner product $\langle\cdot, \cdot\rangle$ on $V$ for which $C=C^{\star}$, where

$$
C^{\star}:=\{y \in V \mid\langle y, x\rangle>0 \text { for all } x \in \operatorname{cl} C\}
$$

is the open dual of $C$.

By the Koecher-Vinberg theorem, there is a one-to-one correspondence between symmetric cones and finite-dimensional real Euclidean Jordan algebras. Indeed, each symmetric cone arises as the set of square elements of such an algebra.

The map $x \mapsto x^{-1}$ taking the Jordan algebra inverse of an element is an involution on the cone of square elements. This map is also antihomogeneous and an antimorphism. It agrees on symmetric cones with Vinberg's *-map, which can be defined more generally.

For more about symmetric cones, see [4].

\section{Proofs}

3.1. Continuity of order antimorphisms. It was shown in [12] that order isomorphisms between cones are necessarily continuous. The same result holds for antimorphisms, with a similar proof.

Lemma 3.1. Let $\phi: C^{\prime} \rightarrow C$ be an order antimorphism between two proper open convex cones. Then, $\phi$ is continuous.

Proof. Let $x_{n}$ be a sequence in $C$ converging to a point $x \in C$. Take some point $p \in C$, and let $\epsilon>0$ be small enough that $x-\epsilon p$ is contained in $C$. So, $x_{n}$ is eventually in the order interval $I_{\epsilon}:=[x-\epsilon p, x+\epsilon p]$, and hence $\phi\left(x_{n}\right)$ is eventually in the interval $I_{\epsilon}^{\prime}:=[\phi(x+\epsilon p), \phi(x-\epsilon p)]$. The latter is a compact set, and so $\phi\left(x_{n}\right)$ converges if it has only one limit point. Consider a limit point $y$. We have $y \in I_{\epsilon}^{\prime}$, and hence $\phi^{-1}(y) \in I_{\epsilon}$. Since $\epsilon$ was arbitrary, this implies that $\phi^{-1}(y)=x$. We conclude that $\phi\left(x_{n}\right)$ converges to $\phi(x)$. 
3.2. Extreme rays. Recall that a convex subset $E$ of a convex set $D$ is said to be an extreme set if the endpoints of any line segment in $D$ are contained in $E$ whenever any interior point of the line segment is. The only zero-dimensional extreme set of the closure of a proper open convex cone $C$ is the origin $\{0\}$. We call the one-dimensional extreme sets of $\operatorname{cl} C$ its extreme rays, and denote the set of them by $\mathcal{R}_{C}$. An extreme vector of $C$ is a non-zero element of an extreme ray of $C$.

The importance of extreme rays when studying antimorphisms is made clear by the following lemma.

Lemma 3.2. Let $\phi: C \rightarrow C^{\prime}$ be an order antimorphism between two proper open convex cones. Let $x$ and $y$ be distinct points in $C$ such that the line through them is parallel to an extreme ray of $C$, Then, the line through $\phi(x)$ and $\phi(y)$ is parallel to an extreme ray of $C^{\prime}$.

Proof. The proof of this lemma is the similar to the proofs in [10] and [3] for the case of order isomorphisms. The main idea is to characterise the line segments parallel to extreme rays as the order intervals that are total. Recall that a total set is one in which every two elements are comparable.

3.3. A characterisation of product cones. There is simple relation between the extreme rays of a product cone and those of its factors.

Proposition 3.3. The extreme rays of a proper open convex cone that can be written as a product $C=C_{1} \times C_{2}$ are given by

$$
\mathcal{R}_{C}=\left\{R \times\{0\} \mid R \in \mathcal{R}_{C_{1}}\right\} \cup\left\{\{0\} \times R \mid R \in \mathcal{R}_{C_{2}}\right\} .
$$

Proof. A set is an extreme set of $\operatorname{cl} C$ if and only if it can be written as the product of an extreme set of $\operatorname{cl} C_{1}$ and an extreme set of $\operatorname{cl} C_{2}$. For such a set to be one-dimensional, it must be the product of a zero-dimensional extreme set of one of the factors and a one-dimensional extreme set of the other.

We wish to characterise irreducible cones in terms of the linear dependencies of their extreme rays. Let $C$ be a proper open convex cone. We say that a set of extreme rays of $C$ is linearly independent if one obtains a linearly independent set when one takes a single non-zero representative of each ray. A set of extreme rays that is not linearly independent is linearly dependent.

Consider now the minimal dependent subsets of the set of extreme rays, that is, the subsets that are linearly dependent but do not contain any proper linearly dependent subset. Minimal dependent sets are also known as a circuits in matroid theory. Define the following equivalence relation between extreme rays $R$ and $R^{\prime}$ in $\mathcal{R}_{C}$. Write $R \sim R^{\prime}$ if either $R=R^{\prime}$ or there exists a minimal dependent subset of $\mathcal{R}_{C}$ containing both. This relation is obviously reflexive and symmetric; its transitivity is more subtle - see Theorem 3.36 of [5]. The next proposition shows that irreducibility of the cone $C$ is equivalent to there being only one equivalence class of elements of $\mathcal{R}_{C}$.

Proposition 3.4. A proper open convex cone $C$ is irreducible if and only if every pair of distinct elements of $\mathcal{R}_{C}$ is contained in a minimal dependent subset of $\mathcal{R}_{C}$.

Proof. First assume that $C=C_{1} \times C_{2}$ is a product cone. Take $R_{1} \in \mathcal{R}_{C_{1}}$ and $R_{2} \in \mathcal{R}_{C_{2}}$. According to Proposition 3.3, the rays $R_{1} \times\{0\}$ and $\{0\} \times R_{2}$ are in $\mathcal{R}_{C}$, and they are clearly distinct. Let $\mathcal{D}$ be a dependent subset of $\mathcal{R}_{C}$ containing $R_{1} \times\{0\}$ and $\{0\} \times R_{2}$ Again from Proposition 3.3, there are sets $\mathcal{D}_{1} \subset \mathcal{R}_{C_{1}}$ and $\mathcal{D}_{2} \subset \mathcal{R}_{C_{2}}$ such that each of 
the elements of $\mathcal{D}$ is either of the form $R \times\{0\}$ with $R \in \mathcal{D}_{1}$, or $\{0\} \times R$ with $R \in \mathcal{D}_{2}$. Note that $R_{1} \in \mathcal{D}_{1}$ and $R_{2} \in \mathcal{D}_{2}$. For each $R \in \mathcal{D}_{1} \cup \mathcal{D}_{2}$, choose a non-zero representative $x_{R}$. We have, for some coefficients $\left\{\lambda_{R}\right\} ; R \in \mathcal{D}_{1} \cup \mathcal{D}_{2}$, not all of which are zero,

$$
\sum_{R \in \mathcal{D}_{1}} \lambda_{R}\left(x_{R}, 0\right)+\sum_{R \in \mathcal{D}_{2}} \lambda_{R}\left(0, x_{R}\right)=0 .
$$

Looking at the components separately, we see that each of the two sums is zero. So, $\mathcal{D}$ is not a minimal dependent subset of $\mathcal{R}_{C}$.

Now assume that there are a pair of elements of $\mathcal{R}_{C}$ that are not contained in a minimal dependent set. So, there are at least two equivalence classes of rays in $\mathcal{R}_{C}$ with respect to the relation $\sim$. Let $\mathcal{D}_{1}$ be one of these equivalence classes, and let $\mathcal{D}_{2}$ be its complement. Define the cone $C_{1}$ to be the interior of the cone generated by the elements of $\mathcal{D}_{1}$, and the cone $C_{2}$ to be the interior of the one generated by the elements of $\mathcal{D}_{2}$. Also, let $V_{1}:=\operatorname{lin} \mathcal{D}_{1}$ and $V_{2}:=\operatorname{lin} \mathcal{D}_{2}$ be the linear subspaces in which these cones lie. Clearly, $C=C_{1}+C_{2}$.

Choose a basis $B_{1}$ of $V_{1}$ consisting of extreme vectors of $C$, each one lying in one of the extreme rays in $\mathcal{D}_{1}$. Likewise, choose a basis $B_{2}$ of $V_{2}$ consisting extreme vectors each lying in one of the extreme rays in $\mathcal{D}_{2}$. Observe that no subset of $B_{1} \cup B_{2}$ is a minimal dependent set. It follows that $B_{1} \cup B_{2}$ is independent. We conclude that $V_{1} \cap V_{2}=\{0\}$. Thus, $C$ is the product of $C_{1}$ and $C_{2}$.

3.4. Antimorphisms on product cones. In this section, we will prove Theorem 1.3, that is, that order antimorphisms on product cones respect the product structure.

Let $C$ be a proper open convex cone. We topologise it set of extreme rays $R_{C}$ by taking the Kuratowski-Painlevé topology on the set of closed sets of $V$. In this topology, a sequence of closed sets $\left(D_{n}\right)_{n \in \mathbb{N}}$ is said to converge to a closed set $D$ if each of the following hold:

- for each $x \in D$, there exists $x_{n} \in D_{n}$ for $n$ large enough, such that $\left(x_{n}\right)_{n}$ converges to $x$.

- if $\left(D_{n_{k}}\right)_{k \in \mathbb{N}}$ is a subsequence of the sequence of sets and $x_{k} \in D_{n_{k}}$ for each $k \in \mathbb{N}$, then convergence of $\left(x_{k}\right)_{k \in \mathbb{N}}$ to $x$ implies that $x \in D$.

Note that a sequence of rays starting at the origin converge in this topology if and only if their directions converge. Also, a sequence of closed line segments converge if and only if both sequences of endpoints converge, after possible reversing some of the line segments.

Let $\phi: C \rightarrow C^{\prime}$ be an antimorphism from $C$ to another proper open convex cone $C^{\prime}$. Recall that by Lemma 3.2 every line segment in $C$ that is parallel to an extreme ray of $C$ is mapped by $\phi$ to a line segment parallel to an extreme ray of $C^{\prime}$. Fix an extreme ray $R \in \mathcal{R}_{C}$ and consider the map $\Xi_{R}: C \rightarrow \mathcal{R}_{C^{\prime}}$ defined so that, for each $x \in C$, line segments in $C$ parallel to $R$ that pass through $x$ are mapped to line segments parallel to $\Xi_{R}(x)$.

Lemma 3.5. For each $R \in \mathcal{R}_{C}$, the map $\Xi_{R}$ is continuous.

Proof. Let $x_{n}$ be a sequence in $C$ converging to $x \in C$. Take a closed line segment $L \subset C$ that starts at $x$, and for each $n \in \mathbb{N}$, let $L_{n}$ be the line segment obtained from $L$ by parallel transporting from $x$ to $x_{n}$. For $n$ large enough, $L_{n}$ lies in $C$. Observe that $L_{n}$ converges to $L$ in the Kuratowski-Painlevé topology, as $n$ tends to infinity. By Lemma $3.1, \phi$ is a homeomorphism, and so we get that $\phi\left(L_{n}\right)$ converges to $\phi(L)$. We deduce that $\Xi_{R}\left(x_{n}\right)$ converges to $\Xi_{R}(x)$. 
We can now prove Theorem 1.3. For simplicity, we start with the case of two factors.

Lemma 3.6. Let $\phi: C \rightarrow C^{\prime}$ be an order antimorphism between two proper open convex cones. Assume that $C$ has a product structure: $C=C_{1} \times C_{2}$. Then, $C^{\prime}$ can also be written as the product of two cones: $C^{\prime}=C_{1}^{\prime} \times C_{2}^{\prime}$. Moreover, $\phi$ respects the product structure, that is, there exist order antimorphisms $\phi_{1}: C_{1} \rightarrow C_{1}^{\prime}$ and $\phi_{2}: C_{2} \rightarrow C_{2}^{\prime}$ such that

$$
\phi\left(\left(x_{1}, x_{2}\right)\right)=\left(\phi_{1}\left(x_{1}\right), \phi_{2}\left(x_{2}\right)\right), \quad \text { for all } x_{1} \in C_{1} \text { and } x_{2} \in C_{2} .
$$

Proof. Take an extreme ray $R^{\prime}$ of $C^{\prime}$, and define the map $\Xi_{R^{\prime}}: C^{\prime} \rightarrow \mathcal{R}_{C}$ so that, for each $x \in C^{\prime}$, any line segment in $C^{\prime}$ parallel to $R^{\prime}$ that passes through $x$ is mapped by $\phi^{-1}$ to a line segment parallel to $\Xi_{R^{\prime}}(x)$. The map $\Xi_{R^{\prime}}$ is continuous by Lemma 3.5. So, since there is a continuous path between any two points in $C^{\prime}$, there must be one between any two points in the image $\Xi_{R^{\prime}}\left(C^{\prime}\right)$. Given the form of the extreme rays of $C$ described in Proposition 3.3, we deduce that every ray in $\Xi_{R^{\prime}}\left(C^{\prime}\right)$ must be associated to the same factor of $C$, in other words, $\Xi_{R^{\prime}}\left(C^{\prime}\right)$ must be a subset of either $\mathcal{R}_{C_{1}}$ or $\mathcal{R}_{C_{2}}$.

Let $\mathcal{R}_{1}$ be the set of those $R^{\prime} \in \mathcal{R}_{C^{\prime}}$ for which the former is the case, and $\mathcal{R}_{2}$ be the set of those for which the latter is. Denote by $C_{1}^{\prime}$ and $C_{2}^{\prime}$, respectively, the interiors of the cones generated by these two sets of rays, and $V_{1}^{\prime}$ and $V_{2}^{\prime}$, respectively, the linear spaces generated. Clearly, $C^{\prime}=C_{1}^{\prime}+C_{2}^{\prime}$ and $V^{\prime}=V_{1}^{\prime}+V_{2}^{\prime}$.

Let $v \in V^{\prime}$, and suppose we can write

$$
v=\sum_{i=0}^{m} \lambda_{i} x_{i}=\sum_{i=0}^{n} \mu_{i} y_{i},
$$

where the $x_{i}$ are extreme vectors of $C_{1}^{\prime}$, the $y_{i}$ are extreme vectors of $C_{2}^{\prime}$, and the $\lambda_{i}$ and $\mu_{i}$ are non-zero coefficients. Consider the partial sums $X_{j}:=\sum_{i=0}^{j} \lambda_{i} x_{i}$, with $0 \leq j \leq m$, and $Y_{j}:=\sum_{i=0}^{j} \mu_{i} y_{i}$, with $0 \leq j \leq n$. By choosing a point $z \in C^{\prime}$ far enough away from the origin, we can ensure that $z+X_{j}$ and $z+Y_{j}$ are in $C^{\prime}$ for all $j$.

Since, for each $j$, the points $z+X_{j}$ and $z+X_{j+1}$ differ by multiple of an extreme vector of $C_{1}^{\prime}$, their images $\phi^{-1}\left(z+X_{j}\right)$ and $\phi^{-1}\left(z+X_{j+1}\right)$ differ by a multiple of an extreme vector of $C_{1}$. Summing, we obtain that $\phi^{-1}(z)-\phi^{-1}(z+v)$ is contained in $\operatorname{lin} C_{1}$. Similar reasoning shows that this vector is also contained in $\operatorname{lin} C_{2}$. We deduce that it is zero, which implies that $v$ is zero, since $\phi^{-1}$ is bijective. We have proved that $V_{1}^{\prime} \cap V_{2}^{\prime}=\{0\}$. In conclusion, $C^{\prime}$ is the product of $C_{1}^{\prime}$ and $C_{2}^{\prime}$.

Let $w:=\left(w_{1}, w_{2}\right)$ and $z:=\left(z_{1}, z_{2}\right)$ be points in $C^{\prime}$ sharing a first component, that is, $w_{1}=z_{1}$. So, $z=w+\sum_{j} \lambda_{j} x_{j}$, where the $x_{j}$ are a finite number of extreme vectors of $C_{2}^{\prime}$, and the $\lambda_{j}$ are non-zero coefficients. Using a similar argument to before, we get that the difference between $\phi^{-1}(w)$ and $\phi^{-1}(z)$ lies in lin $C_{2}$, and hence these pre-images have the same first component. Similarly, if $w$ and $z$ have the same second component, then the same is true for $\phi^{-1}(w)$ and $\phi^{-1}(z)$.

So there exist maps $\nu_{1}: C_{1}^{\prime} \rightarrow C_{1}$ and $\nu_{2}: C_{2}^{\prime} \rightarrow C_{2}$ such that $\phi^{-1}\left(\left(w_{1}, w_{2}\right)\right)=$ $\left(\nu_{1}\left(w_{1}\right), \nu_{2}\left(w_{2}\right)\right)$, for all $w_{1} \in C_{1}^{\prime}$ and $w_{2} \in C_{2}^{\prime}$, The injectivity and surjectivity of $\nu_{1}$ and $\nu_{2}$ follow easily from the corresponding properties of $\phi^{-1}$. Define $\phi_{1}:=\nu_{1}^{-1}$ and $\phi_{2}:=\nu_{2}^{-1}$. The map $\left(x_{1}, x_{2}\right) \mapsto\left(\phi_{1}\left(x_{1}\right), \phi_{2}\left(x_{2}\right)\right)$ is the inverse of $\phi^{-1}$, and hence equal to $\phi$.

Proof of Theorem 1.3. We treat the factors of $C$ one-by-one, applying Lemma 3.6 each time. 
3.5. Simplicial slices. In the case of order isomorphisms, one can show that a pair of line segments parallel to the same extreme ray of the cone remain parallel under the mapping. This is not true in general for order antimorphisms, but we will see that it is true when one restricts to certain subcones that we call simplicial slices.

An (open) simplicial cone is a cone that is linearly isomorphic to int $\mathbb{R}_{+}^{n}$, for some $n \geq 1$. In other words, it is a cone over an open simplex. An open cone is a simplicial cone if and only if it is a lattice with respect to its own ordering; see [6, page 221].

Let $C$ be a proper open convex cone in a finite-dimensional linear space $V$. We say that a cone $S \subset V$ is a simplicial slice of $C$ if $S$ is a non-empty simplicial cone that can be written as $S=L \cap C$, where $L$ is a linear subspace of $V$, and each of the extreme rays of $S$ is an extreme ray of $C$.

Observe that if $S$ is a simplicial slice of $C$, then the partial order on $S$ coming from the cone structure of $S$ is the same as the partial order inherited from $C$.

The following is Proposition 3 of [10].

Proposition 3.7. Let three pairwise disjoint half-lines be given such that every point of each lies on a line that meets the other two. Then, all three half-lines are parallel to one plane. Moreover, if two of them lie in one plane, then the third also lies in that plane.

Recall that, for each extreme ray $R \in \mathcal{R}_{C}$, we have defined the map $\Xi_{R}: C \rightarrow \mathcal{R}_{C^{\prime}}$ so that, for each $x \in C$, any line segment in $C$ that passes through $x$ and is parallel to $R$ is mapped to a line segment parallel to $\Xi_{R}(x)$.

Lemma 3.8. Let $\phi: C \rightarrow C^{\prime}$ be an order antimorphism between two proper open convex cones in a finite-dimensional linear space. Let $S$ be a simplicial slice of $C$, and let $R$ be an extreme ray of $S$. Then, $\Xi_{R}$ is constant on $S$.

Proof. It suffices to show that $\Xi_{R}\left(x_{1}\right)=\Xi_{R}\left(x_{2}\right)$ for every pair of distinct points $x_{1}$ and $x_{2}$ in $S$ such that $x_{2}-x_{1}$ is an extreme vector of $S$. This is clearly true if $x_{2}-x_{1}$ lies in $R$, so assume the contrary. Denote by $R^{\prime}$ the extreme ray of $S$ in which $x_{2}-x_{1}$ lies.

Take the midpoint $x_{3}:=\left(x_{1}+x_{2}\right) / 2$ of the two points. Let $L_{1}$ be the open line segment parallel to $R$ with one endpoint at $x_{1}$ and the other on the boundary of $C$. The endpoint on the boundary is also on the boundary of $S$, since $S$ is a simplicial slice. Define the line segments $L_{2}$ and $L_{3}$ in the same way, starting from points $x_{2}$ and $x_{3}$, respectively.

These three line segments are clearly pairwise disjoint. Because $S$ is simplicial, every point lying on one of the segments lies on a line parallel to $R^{\prime}$ that meets the other two segments.

Consider the images under $\phi$ of the line segments; see figure 1. Each is a half line starting at $\phi\left(x_{1}\right), \phi\left(x_{2}\right)$, or $\phi\left(x_{3}\right)$, and parallel to $\Xi_{R}\left(x_{1}\right), \Xi_{R}\left(x_{2}\right)$, or $\Xi_{R}\left(x_{3}\right)$, respectively.

From the properties of $L_{1}, L_{2}$, and $L_{3}$ stated in the previous paragraph, we get using Lemma 3.2 that their images $\phi\left(L_{1}\right), \phi\left(L_{2}\right)$, and $\phi\left(L_{3}\right)$ satisfy the assumptions of Lemma 3.7. Therefore, these images are all parallel to some plane. This means that $\Xi_{R}\left(x_{1}\right), \Xi_{R}\left(x_{2}\right)$, and $\Xi_{R}\left(x_{3}\right)$ are co-planar, and hence at least two of them must be identical. We conclude that two of the half lines $\phi\left(L_{1}\right), \phi\left(L_{2}\right)$, and $\phi\left(L_{3}\right)$ are parallel and therefore lie in a plane. Applying Lemma 3.7 again, we get that all three of them lie in the plane.

This plane also contains the line segment $\phi(M)$, where $M$ is the line segment between $x_{1}$ and $x_{2}$. Here we are using Lemma 3.2, to get that $\phi(M)$ is a line segment parallel to an extreme ray $\Xi_{R^{\prime}}\left(x_{1}\right)$ of $C^{\prime}$. So, the extreme rays $\Xi_{R}\left(x_{1}\right), \Xi_{R}\left(x_{2}\right)$, and $\Xi_{R^{\prime}}\left(x_{1}\right)$ are 

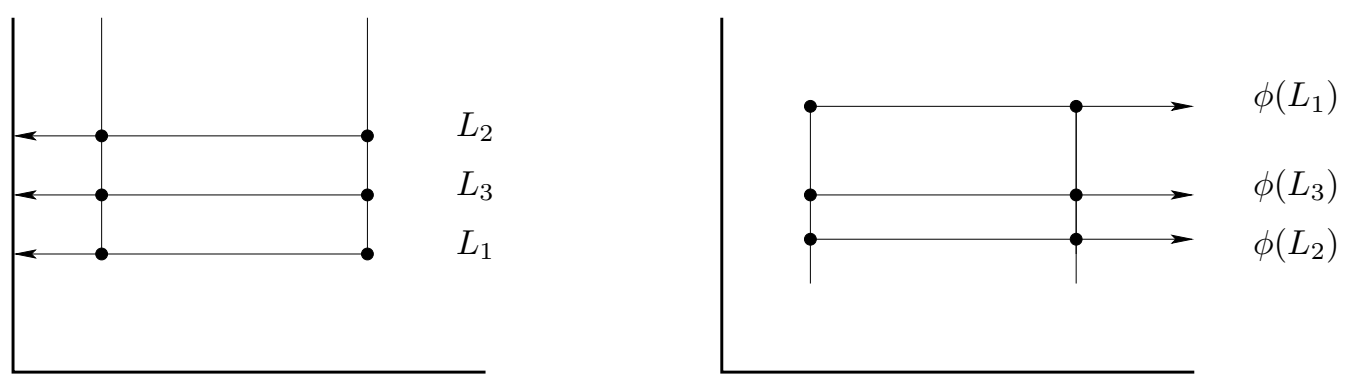

Figure 1. Diagram for the proof of Lemma 3.8.

co-planar, and hence at least two of them are equal, necessarily the first two, because $\phi(M)$ is not parallel to either $\phi\left(L_{1}\right)$ or $\phi\left(L_{2}\right)$.

Next we show that simplicial slices are mapped to simplicial slices.

Lemma 3.9. Let $\phi: C \rightarrow C^{\prime}$ be an order antimorphism between two proper open convex cones in a finite-dimensional linear space. Let $S$ be a simplicial slice of $C$. Then, there is a simplicial slice $S^{\prime}$ of $C^{\prime}$ such that $\phi(S)=S^{\prime}$.

Proof. Denote by $\left\{R_{i}\right\}$ the extreme rays of the simplicial slice $S$. By Lemma 3.8, for each $i$, there is some extreme ray $R_{i}^{\prime}$ of $C^{\prime}$ such that each line segment in $S$ parallel to the ray $R_{i}$ is mapped by $\phi$ to a line segment parallel to $R_{i}^{\prime}$. Let $U$ be the linear hull of the union of the rays $\left\{R_{i}^{\prime}\right\}$. Take a point $x \in S$, and define the affine space $A:=\phi(x)+U$.

Let $y$ be any point in $S$. There exists a finite sequence of points $x_{0}, \ldots, x_{n}$ such that $x_{0}=x, x_{n}=y$, and, for each $j \in\{0, \ldots, n-1\}$, the line segment between $x_{j}$ and $x_{j+1}$ is parallel to one of the $R_{i}$. So, for each $j$, the line segment between $\phi\left(x_{j}\right)$ and $\phi\left(x_{j+1}\right)$ is parallel to one of the $R_{i}^{\prime}$. We conclude that all the $\phi\left(x_{j}\right)$ lie in $A$, in particular $\phi(y)$. So, $\phi(S)$ is contained in $A \cap C^{\prime}$.

The reverse inclusion is proved in a similar manner.

Let $z \in S$ and consider the sequence of points $z_{n}:=n z$. Given any point $w$ of $C$, the sequence $z_{n}$ is eventually greater than $w$. So, for any $w^{\prime}$ in $C^{\prime}$, the sequence $\phi\left(z_{n}\right)$ is eventually less than $w^{\prime}$. It follows that $\phi\left(z_{n}\right)$ converges to the origin. We have shown that the origin is in the affine space $A$. So, $A$ is actually equal to the linear subspace $U$ of $V^{\prime}$.

Let $S^{\prime}:=U \cap C^{\prime}$, which we have seen is equal to $\phi(S)$. Observe that the order on $S^{\prime}$ inherited from $C^{\prime}$ is the same as the order coming from the closed cone $\mathrm{cl} S^{\prime}$. So, the restriction of $\phi$ to $S$ is an order antimorphism between $S$ and $S^{\prime}$. Since $S$ with its cone order is a lattice, so also is $S^{\prime}$. This implies that $S^{\prime}$ is a simplicial cone.

Each ray $R_{i}^{\prime}$ is in $\mathrm{cl} S^{\prime}$ and is an extreme ray of $C^{\prime}$. Hence, each of these rays is also an extreme ray of $S^{\prime}$. But the dimension of $U$ is at most the number of rays $\left\{R_{i}^{\prime}\right\}$. So, $S^{\prime}$ can have at most this number of extreme rays. This means that the set of extreme rays of $S^{\prime}$ is exactly the set of rays $\left\{R_{i}^{\prime}\right\}$. It is now clear that every extreme ray of $S^{\prime}$ is an extreme ray of $C^{\prime}$. This completes the proof that $S^{\prime}$ is a simplicial slice of $C^{\prime}$.

We will need that there are many simplicial slices. In fact, every extreme ray of a cone is an extreme ray of some simplicial slice.

Lemma 3.10. Let $R$ be extreme ray of a proper open convex cone $C$. Then, there exists a simplicial slice $S$ of $C$ such that $R$ is an extreme ray of $S$. 
Proof. Let $r \in R \backslash\{0\}$. Choose any point $q$ in $C$. The linear span of $r$ and $q$ defines a two-dimensional subspace of $V$, the intersection of which with $C$ is a two-dimensional cone having $R$ as a boundary component. The other boundary component is a ray that lies in the boundary of $C$ but is not necessarily extreme. Let $E$ be the smallest proper extreme set of $\operatorname{cl} C$ that contains this ray.

Observe that the linear span of $R \cup E$ intersects $C$; indeed, it contains $q$. Choose a basis $\mathcal{E}$ of $\operatorname{lin} E$ consisting of extreme vectors of $C$. We have that lin $\mathcal{E}$ does not intersect $C$, whereas $\operatorname{lin}(\{r\} \cup \mathcal{E})$ does.

Consider any element $r^{\prime}$ of $\mathcal{E}$. If $\operatorname{lin}\left(\{r\} \cup \mathcal{E} \backslash\left\{r^{\prime}\right\}\right)$ intersects $C$, then remove $r^{\prime}$ from $\mathcal{E}$. Continue removing elements of $\mathcal{E}$ one-by-one in this way as long as it is possible to find one satisfying this condition.

Write $\mathcal{E}^{\prime}:=\{r\} \cup \mathcal{E}$. The procedure of the previous paragraph results in a situation where $\operatorname{lin} \mathcal{E}^{\prime}$ intersects $C$, but $\operatorname{lin}\left(\mathcal{E}^{\prime} \backslash\left\{r^{\prime}\right\}\right)$ does not, for any $r^{\prime} \in \mathcal{E}$. In particular, $\mathcal{E}^{\prime}$ is a linearly independent set of vectors.

Let $S$ be the relatively open simplicial cone generated by $\mathcal{E}^{\prime}$. The extreme rays of $S$ are the rays $\left\{\lambda r^{\prime} \mid \lambda \geq 0\right\} ; r^{\prime} \in \mathcal{E}^{\prime}$. Each of these is also an extreme ray of $C$.

Define $H:=\operatorname{lin} \mathcal{E}^{\prime} \cap \operatorname{cl} C$. Since $H$ is convex and contains $\mathcal{E}^{\prime}$, we have $\operatorname{cl} S \subset H$, where the closure is taken in the space lin $\mathcal{E}^{\prime}$. Furthermore, for each $e \in \mathcal{E}^{\prime}$, the linear space $\operatorname{lin}\left(\mathcal{E}^{\prime} \backslash\{e\}\right)$ is a tangent hyperplane to $H$ in the linear space lin $\mathcal{E}^{\prime}$. It follows that $H \subset \operatorname{cl} S$. We have shown that $\operatorname{cl} S=H$. We conclude that $S=\operatorname{lin} \mathcal{E}^{\prime} \cap C$.

3.6. The antihomogeneity defect map. Suppose we are given a bijection $\phi$ between two proper open convex cones $C$ and $C^{\prime}$. For each $\lambda>0$, define the antihomogeneity defect map:

$$
\Delta_{\lambda}:=\phi^{-1} \circ M_{\lambda} \circ \phi \circ M_{\lambda} .
$$

Here $M_{\lambda}$ means multiplication by the scalar $\lambda$. The map $\Delta_{\lambda}$ measures how far away from being antihomogeneous the map $\phi$ is. Indeed, if $\phi$ is antihomogeneous, then $\Delta_{\lambda}$ is the identity map. If $\phi$ is an order antimorphism, then $\Delta_{\lambda}$ is an order isomorphism. In any case, $\Delta_{\lambda}$ is a bijection from $C$ to itself.

We wish to show that, in the case of antimorphisms on irreducible cones, the defect map is the identity. Our strategy will be to first show that it is a homothety. Recall that a homothety is a linear map of the form $x \mapsto \alpha x$, with $\alpha \in \mathbb{R}$.

Lemma 3.11. Let $C$ be an irreducible proper open convex cone in a finite-dimensional vector space $V$, and let $L: V \rightarrow V$ be linear map such that every extreme vector of $C$ is an eigenvector of $L$. Then, $L$ is a homothety.

Proof. The conclusion is trivial if $C$ is one-dimensional, so assume the contrary.

Let $e_{1}$ and $e_{2}$ be extreme vectors of $C$ that are not scalar multiples of one another. Since $C$ is irreducible, there exists by Proposition 3.4 a minimal dependent set $E$ of extreme vectors of $C$ containing both $e_{1}$ and $e_{2}$. Write $E=:\left\{e_{1}, e_{2}, \ldots, e_{n}\right\}$. Because $E$ is minimal dependent, we have, for some non-zero real coefficients $\left\{a_{i}\right\}$,

$$
e_{1}=a_{2} e_{2}+\cdots+a_{n} e_{n} .
$$

For each $i$, let $\lambda_{i}$ be the eigenvalue of $e_{i}$ with respect to $L$. Applying $L$ to the above equation, we get

$$
\lambda_{1} e_{1}=\lambda_{2} a_{2} e_{2}+\cdots+\lambda_{n} a_{n} e_{n}
$$


So, since the vectors $e_{2}, \ldots, e_{n}$ are linearly independent, we must have $\lambda_{1}=\lambda_{2}=\cdots=$ $\lambda_{n}$. We deduce that all extreme vectors of $C$ have the same eigenvalue. Since there is a basis of $V$ of such vectors, this proves the lemma.

The following lemma explores further the relation between the defect map and the homogeneity of $\phi$.

Lemma 3.12. Let $\phi: C \rightarrow C^{\prime}$ be an order antimorphism between two proper open convex cones. If the defect map $\Delta_{\lambda}$ is a homothety for each $\lambda>0$, then $\phi$ is homogeneous of some degree $\alpha \in \mathbb{R}$.

Proof. Let $\lambda$ and $\lambda^{\prime}$ be positive real numbers. Since $\Delta_{\lambda}$ is linear, it commutes with multiplication by scalars. Therefore,

$$
\Delta_{\lambda^{\prime}} \Delta_{\lambda}=\phi^{-1} \circ M_{\lambda^{\prime}} \circ \phi \circ\left(\phi^{-1} \circ M_{\lambda} \circ \phi \circ M_{\lambda}\right) \circ M_{\lambda^{\prime}}=\Delta_{\lambda^{\prime} \lambda} .
$$

Since, for each $\lambda>0$, the defect map is a homothety, we can write it $\Delta_{\lambda}(y)=\mu(\lambda) y$ for all $y \in C$, with $\mu(\lambda)$ a positive scalar. Consider the function $f: \mathbb{R} \rightarrow \mathbb{R}$, defined by

$$
f(x):=\log \circ \mu \circ \exp (x) .
$$

From what we have just seen, $f$ obeys the functional equation $f\left(x^{\prime}+x\right)=f\left(x^{\prime}\right)+f(x)$, for $x^{\prime}$ and $x$ in $\mathbb{R}$. Moreover, $\phi$ is continuous by Lemma 3.1, and so $f$ is continuous. We deduce that there exists $c \in \mathbb{R}$ such that $f(x)=c x$, for all $x \in \mathbb{R}$; see [7, Theorem 1.1].

This implies that $\mu(\lambda)=\lambda^{c}$, for all $\lambda>0$. Therefore, $\lambda \phi(y)=\phi\left(\lambda^{c-1} y\right)$, for all $\lambda>0$ and $y \in C$. This gives an absurdity if $c$ equals 1 , so evidently this is not the case. We deduce that $\phi$ is homogeneous of degree $1 /(c-1)$.

3.7. Differentiability of antimorphisms. In this section, we establish a couple of lemmas that together show that if there exists an order antimorphism between two cones that is homogeneous of some degree, then the cones are linearly isomorphic. This is accomplished by first showing that the map is Lipschitz with respect to the Thompson metric on the cones, and then using Rademacher's theorem.

Recall that the Thompson metric on a cone $C$ is defined in the following way:

$$
d_{C}(x, y):=\log \max \left\{M_{C}(x, y), M_{C}(y, x)\right\},
$$

where the gauge is

$$
M_{C}(x, y):=\inf \{\lambda>0 \mid x \leq \lambda y\} .
$$

The following result appears in [11, Proposition 1.5].

Lemma 3.13. Let $\phi: C \rightarrow C^{\prime}$ be an antitone map between two cones that is homogeneous of degree $-\alpha$, with $\alpha>0$. Then, $\phi$ is $\alpha$-Lipschitz with respect to the Thompson metric, that is,

$$
d_{C^{\prime}}(\phi(x), \phi(y)) \leq \alpha d_{C}(x, y), \quad \text { for all } x \text { and } y \text { in } C .
$$

Proof. Write $r:=d_{C}(x, y)$. So, $M_{C}(x, y)$ is less than or equal to $\exp r$, which implies that $x \leq \exp (r) y$. Using the properties of $\phi$, we get $\phi(x) \geq \exp (-\alpha r) \phi(y)$. Therefore $M_{C^{\prime}}(\phi(y), \phi(x)) \leq \exp (\alpha r)$. A similar expression holds when $x$ and $y$ are interchanged. The conclusion follows.

Rademacher's theorem states that a Lipschitz map from an open subset of a Euclidean space to another Euclidean space is differentiable almost everywhere, that is, differentiable everywhere except on a set of measure zero. 
Lemma 3.14. Let $\phi: C \rightarrow C^{\prime}$ be an order antimorphism between two proper open convex cones in a finite-dimensional linear space. If $\phi$ and $\phi^{-1}$ are Lipschitz in the Thompson metric, then there exists a linear isomorphism $L: C^{\prime} \rightarrow C$.

Proof. The Thompson metric is Lipschitz equivalent to the Euclidean metric on every Thompson-metric ball of finite radius. So we can use Rademacher's theorem to get that $\phi$ is differentiable almost everywhere on each of these balls, and hence almost everywhere on the whole domain. The same is true of $\phi^{-1}$.

So, we can find $x \in C$ such that $\phi$ is differentiable at $x$ and $\phi^{-1}$ is differentiable at $\phi(x)$. Let $L:=-D_{\phi(x)} \phi^{-1}$ be the differential of $\phi^{-1}$ at $\phi(x)$. The differential $D_{x} \phi$ of $\phi$ at $x$ is then $-L^{-1}$.

It follows easily from the antitonicity of $\phi$ and $\phi^{-1}$ that $L^{-1}$ and $L$, respectively, are isotone. Thus, $L$ is a linear isomorphism.

\subsection{The proof of the theorem.}

Proof of Theorem 1.1. By Theorem 1.3, the map $\phi$ respects the product structure of the cone $C$. Thus, it suffices to prove the result for the component of $\phi$ acting on each irreducible factor. By assumption, none of these factors are one-dimensional. So, without loss of generality, we may assume that $C$ is irreducible and not one-dimensional.

Take $\lambda>0$, and consider the antihomogeneity defect map $\Delta_{\lambda}$, defined in section 3.6. This map is an order isomorphism between $C$ and $C^{\prime}$, and hence, since $C$ has no onedimensional factors, must be linear.

Let $R$ be an extreme ray of $C$. By Lemma 3.10, this ray is an extreme ray of some simplicial slice $S$ of $C$. By Lemma 3.9, there is a simplicial slice $S^{\prime}$ of $C^{\prime}$ such that $\phi(S)=S^{\prime}$. Restricted to $S$, the map $\phi$ is an order antimorphism between two simplicial cones, and thus, by Theorem 1.3 takes the form $\phi\left(x_{1}, \ldots, x_{n}\right)=\left(\phi_{1}\left(x_{1}\right), \ldots, \phi_{n}\left(x_{n}\right)\right)$, where $n$ is the dimension of $S$ and $S^{\prime}$. Here we are using coordinates on $S$ and $S^{\prime}$ coming from their respective linear isomorphisms with $(0, \infty)^{n}$. It follows that the defect map of this restriction also respects the product structure of $S$. Moreover it agrees with $\Delta_{\lambda}$ on $S$, and is hence linear. We conclude that $\Delta_{\lambda}\left(x_{1}, \ldots, x_{n}\right)=\left(\alpha_{1} x_{1}, \ldots, \alpha_{n} x_{n}\right)$, for some positive real numbers $\alpha_{1}, \ldots, \alpha_{n}$. This shows that $R$ is mapped to itself by the linear $\operatorname{map} \Delta_{\lambda}$.

Since this is true for every extreme ray of $C$, we deduce from Lemma 3.11 that $\Delta_{\lambda}$ is a homothety. But this holds for every $\lambda>0$, and so by Lemma 3.12 the map $\phi$ is homogeneous of some degree $\alpha \in \mathbb{R}$, that is, it satisfies $\phi(\lambda x)=\lambda^{\alpha} \phi(x)$ for all $\lambda>0$. Since $\phi$ is antitone and bijective, we have $\alpha<0$.

By Lemma 3.13, the map $\phi$ is $-\alpha$-Lipschitz with respect to the Thompson metric. The inverse map $\phi^{-1}$ is also homogeneous, this time of degree $1 / \alpha$, and so, by Lemma 3.13 again, is $-(1 / \alpha)$-Lipschitz.

So, we get from Lemma 3.14 that there exists a linear isomorphism $L$ from $C^{\prime}$ to $C$. Consider the composition $\psi:=L \circ \phi$. This is an order antimorphism from $C$ to itself. Therefore, $\psi^{2}$ is an order isomorphism from $C$ to itself, and is hence linear since $C$ has no one-dimensional factors. But $\psi$ is homogeneous of degree $\alpha$, and so $\psi^{2}$ is homogeneous of degree $\alpha^{2}$. We conclude that $\alpha=-1$.

Proof of Corollary 1.2. Write $C=C_{1} \times \cdots \times C_{n}$ as a product of indecomposable factors. We must show that each factor is symmetric.

Every one-dimensional factor is linearly isomorphic to $(0, \infty)$, and hence symmetric. 
Consider a factor $C_{i}$ that is not one-dimensional. By Theorem 1.3, $\phi$ respects the product structure, and so there is an order antimorphism $\phi_{i}: C_{i} \rightarrow C_{i}^{\prime}$, where $C_{i}^{\prime}$ is a factor of $C^{\prime}$. By Theorem 1.1, the map $\phi_{i}$ is antihomogeneous. It is therefore a gaugereversing map, and hence $C_{i}$ is a symmetric cone by the result of [13].

\section{REFERENCES}

[1] A. D. Alexandrov and V. V. Ovchinnikova. Notes on the foundations of relativity theory. Vestnik Leningrad University, 11:95-110, 1953. (Russian).

[2] Shiri Artstein-Avidan and Vitali Milman. The concept of duality in convex analysis, and the characterization of the Legendre transform. Ann. Math. (2), 169(2):661-674, 2009.

[3] Shiri Artstein-Avidan and Boaz A. Slomka. Order isomorphisms in cones and a characterization of duality for ellipsoids. Selecta Math. (N.S.), 18(2):391-415, 2012.

[4] Jacques Faraut and Adam Korányi. Analysis on symmetric cones. Oxford Mathematical Monographs. The Clarendon Press Oxford University Press, New York, 1994. Oxford Science Publications.

[5] Gary Gordon and Jennifer McNulty. Matroids: a geometric introduction. Cambridge University Press, Cambridge, 2012.

[6] Peter M. Gruber. Convex and Discrete Geometry. Grundlehren der mathematischen Wissenschaften. Springer, 2007.

[7] Pl. Kannappan. Functional equations and inequalities with applications. Springer Monographs in Mathematics. Springer, New York, 2009.

[8] Bas Lemmens, Mark Roelands, and Hent van Imhoff. An order theoretic characterization of spin factors. Q. J. Math., 68(3):1001-1017, 2017.

[9] Walter Noll and Juan Jorge Schäffer. Orders, gauge, and distance in faceless linear cones; with examples relevant to continuum mechanics and relativity. Arch. Rational Mech. Anal., 66(4):345377, 1977.

[10] Walter Noll and Juan Jorge Schäffer. Order-isomorphisms in affine spaces. Ann. Mat. Pura Appl. (4), 117:243-262, 1978.

[11] Roger D. Nussbaum. Hilbert's projective metric and iterated nonlinear maps. Mem. Amer. Math. Soc., 75(391), 1988.

[12] O. S. Rothaus. Order isomorphisms of cones. Proc. Amer. Math. Soc., 17:1284-1288, 1966.

[13] Cormac Walsh. Gauge-reversing maps on cones, and Hilbert and Thompson isometries. Preprint. arXiv: 1312.7871 .

[14] E. C. Zeeman. Causality implies the Lorentz group. J. Mathematical Phys., 5:490-493, 1964.

inRia and CMAP, École Polytechnique, Université Paris-Saclay, 91128 Palaiseau, France E-mail address: cormac.walsh@inria.fr 